\title{
Reconstructing the Study of Human Cognition
}

\author{
Richard W Prather II \\ University of Maryland - College Park \\ Prather1@umd.edu
}

Version: July 5, 2021 


\begin{abstract}
The study of human cognition is a prominent part of psychology and related disciplines. While the modern approach begun during the Cognitive Revolution has been seemingly successful, it is not without concerns. I address five concerns with how human cognition is studied: (1) reliance on homogenous participant samples when trying to generalize behavior to real-world contexts; (2) focus on controlling for or ignoring "extraneous" variables; (3) assumption of a generic human actor instead of a focus on individual and contextual variation; (4) insufficient theory building.

I contend that these concerns are deeply connected and that the solution is a significant change in how we study human cognition, similar in scope to the Cognitive Revolution. We need to reconsider the assumption of cognitive universals and how that assumption is built into the norms of the discipline. I propose a reconstruction of how researchers study human cognition by implementing a combination of methodological approaches and theoretical positions. These combined approaches (1) integrate human heterogeneity, (2) consider human behavior in context, (3) incorporate multiple levels of analysis and non-cognitive factors, (4) focus not only on averaged behavior but variation across individuals and context, (5) create theory that combines cognition and context.
\end{abstract}

Keywords: metascience, review, cognition, intersectionality 
RECONSTRUCTING COGNITION

\title{
What are the key concerns in the study of human cognition?
}

\author{
"What's more important, the flower or the soil that grow it?"
}

-B. Butcher

Over 60 years past the Cognitive Revolution, there is now a lot of work to look back on to gauge the progress or lack thereof in the field. Now is an excellent time for additional reconsideration of the norms, assumptions, and common approaches in cognitive research. I do not wish to be prescriptive about defining cognitive research. Cognition has ambiguous boundaries that overlap with human affect, social cognition, and other manner of mental processes and behavior. This paper is aimed at researchers who are interested in the topics typically associated with cognition: memory, language, attention, problem-solving, learning, perception, and numerical cognition, among others.

Recent interest in meta-science has led to a proliferation of papers addressing cognitive research's current state and how it may be improved. Work has considered the norms around statistical analysis (e.g., Dienes, 2011), method registration (e.g., Nosek, Ebersole, DeHaven, \& Mellor, 2018), sharing of data and materials (Martone, Garcia-Castro, \& VandenBos, 2018), large-scale cooperative studies (Frank et al., 2017; Moshontz et al., 2018), participant sample diversity (e.g., Roberts, Bareket-Shavit, Dollins, Goldie, \& Mortenson, 2020; Rowley \& Camacho, 2015 ) and the relationship between theory and experiments (Devezer, Navarro, Vandekerckhove, \& Buzbas, 2020; van Rooij \& Baggio, 2020).

I highlight several key concerns with cognitive research that are closely related. I contend that these concerns are fundamentally related to the same set of basic assumptions underlying much of cognitive research. Addressing these concerns will require revisiting these assumptions and norms and reconstructing cognitive research as a field. In part this discussion is about methods and approaches used in cognitive work. It is also about the kinds of scientific questions we try to answer. Though these issues have been addressed individually, it is important to demonstrate that these concerns all point to a fundamental fault in how cognition is currently studied; the assumption of cognitive universals. Cognitive researchers often operate from an assumption of universality, which licenses them to focus on WEIRD participants, ignore context, make general claims, focus on averaged behavior, and not make complex theories that acknowledge the complexity of how cognition is shaped by the world. It may be necessary for cognitive researchers to reconsider what their true object of study is. The object may not be some universal cognition. The correct object is the dynamic and iterative interaction between cognition and context.

The first concern is the reliance on homogenous population samples in order to make generalizations about humans. This is sometimes referred to as the WEIRD (Western, Educated, Industrialized, Rich, Democratic) problem (e.g., Henrich, Heine, \& Norenzayan, 2010). Second, the focus on controlling for or ignoring "extraneous" variables. Third, the assumption of a generic human actor based on averaged data 
instead of focusing on individual differences across a diverse set of humans or acknowledging different distributions of behavior for populations with differing contexts. Fourth, the lack of theory building in favor of cataloging effects.

These concerns are not entirely new, in fact we can see that some similar issues in psychology have been broached previously around the use of individual differences and the effect of context on behavior (Cronbach, 1957; Jenkins, 1979; Roediger, 2008); theoretical work on the difficulty of answering scientific question in isolation (Newell, 1973); the usefulness, or lack thereof of psychological research (Banaji \& Crowder, 1989; Neisser, 1978). In this sense the current manuscript is part of a long tradition of theoretical and meta-scientific work concerning the study of human cognition.

The aforementioned issues represent a long tension in the study of human cognition that some researchers have grappled with, one way or another. On the other hand, many of these issues remain. It may not be that researchers are totally unaware, but more that there need to be other options that the status quo approach.

Of course, research on human cognition is not universally approached with the same manner across all areas. For example work in intelligence and applied cognitive psychology may have a more prominent norm of addressing individual differences (e.g., Tourva \& Spanoudis, 2020) and some aspects of participant context (e.g., Daniele, 2021; Young et al., 2021). Research in ecological psychology (Heft, 2013) considers the dynamic interaction with the broader physical context. Crosscultural work can also demonstrate how cultural context affects basic cognition (e.g., Chua, Boland, \& Nisbett, 2005; Kitayama, Duffy, Kawamura, \& Larsen, 2003; Kuwabara \& Smith, 2012).

In the current we raise two important points. One is the integration of context in to cognition work, not simply as an interesting effect as we see with many measured variables, but as fundamental a part of cognition as the mental processes. While there are certainly cases of cognitive researchers focusing on some part of the participants context, such as the recent interest in socioeconomic status, context is largely framed as an interesting side note, and not a fundamental part of cognition. Part of characterizing context is characterizing aspects of who the participants are, their developmental, cultural and societal context. While I do not content that no cognitive researchers do. I introduce intersectionality (Crenshaw, 1989) and more recently quantitative intersectionality (Else-Quest \& Hyde, 2016b, 2016a) as avenues for a more comprehensive consideration of participants' context.

Focus on participants context does not mean that potential generalizations are counter to inclusive cognitive research. There likely are some aspects of cognition that will generalize across humans. There are likely also many aspects that can vary somewhat depending on participants developmental, cultural and societal context. The goal here isn't necessarily to exclusively focus on differences, so much as to elevate context as a natural part of experience driven human variation. To be able to do this in a comprehensive fashion requires a thorough characterization of people's developmental, cultural and societal context.

I do not expect that the concerns or potential solutions in the current work to be entirely novel. It is clear that various researchers interested in cognition and human behavior have been thinking about these issues separately. I do think it is 
important to consider that the concerns are not isolated problems but point to broad concerns about how cognitive research can be rigourous, inclusive, emancipatory. In a sense I am acting as an orchestra conductor attempting to get different groups to play together to combine to construct something together greater than any single group could.

\section{Generalizing from WEIRD participants}

The articulation of general principles of cognition that apply to all humans is the often unstated but understood goal of cognitive research (e.g., Simons, Shoda, \& Lindsay, 2017). The WEIRD problem is the idea is that homogenious participant samples from WEIRD contexts are significantly over-represented in psychological research. Studies have pointed out that there are overrepresentations of participants in WEIRD contexts, particularly participants from the USA (Cheon, Melani, \& Hong, 2020; Henrich et al., 2010; Rad, Martingano, \& Ginges, 2018). The amount of this skew in the participant sample varies across areas of psychology. Cognitive research may be worse compared to social (Roberts et al., 2020). Either way, homogenous participant groups are a problem that cognitive research seems to have. When a cognitive study is done in a lab in the Psychology Building of a North American university with primarily white middle-class undergraduate students between 18 and 22 years old, to whom are the results going to generalize? How much can cognitive researchers hold on to the idea that a population that is largely wealthy, white college students can be used as the benchmark for human cognition?

Even for 'basic' cognitive processes, there appears to be variation across different populations-a great example of this in cognitive processes involved in perception. The well-known Muller-Lyer illusion (Figure 2) results vary across different populations of people when compared to adults in the USA (Segall, Campbell, \& Herskovits, 1966). The variations in the effect may be due to differences in experience with "carpentered corners" that are common in modernized environments. Participants in environments where they experience such corners less would be less likely to display the illusion. In this particular case, adults in the USA are outliers compared to the global population (Henrich, et al., 2010). Additionally in spatial cognition and development of visual search there are variations across cultural contexts (Chua et al., 2005; Kitayama et al., 2003; Kuwabara \& Smith, 2012).

We see similar variation when looking at the development of motoric skills. Researchers have found that experience wearing cushioned running shoes leads to changes in the mechanics a person uses in running (Lieberman et al., 2010). People who do not wear such shoes, of which there are many globally, would not have this change. Research on running using a homogenous WEIRD sample would not generalize to all humans (Henrich et al., 2010). The issue of shoe usage should clarify that the differences that make generalizations difficult are developmental differences based on prior experiences, not some hypothetical underlying genetic difference between populations. If basic motor and perceptual tasks are performed differently due to participants' experience, how confident should cognitive researchers be that cognitive processes readily generalize across all humans? Even basic cognitive processes are subject to the influences of developmental experience. 
Proposals to address the WEIRD problem include recruiting more from heterogeneous non-WEIRD participant groups. The idea is that a more representative sample may generalize better. There are strategies for recruitment in cognitive development (Rowley \& Camacho, 2015), the use of Internet platforms to recruit globally (Buhrmester, Kwang, \& Gosling, 2011), calls for journals to assess participant heterogeneity, perhaps through badges and other nudges (Henrich et al., 2010). Funding agencies have also considered policies that encourage sampling of underrepresented groups. The National Institutes of Health, for example, has a stated policy on the inclusion of women and minorities as participants in research involving human subjects: "The primary goal of this law is to ensure that research findings can be generalizable to the entire population. Additionally, the statute requires clinical trials to be designed to provide information about differences by sex/gender, race, and/or ethnicity." (National Institutes of Health, 2017),

It may not be feasible to aim for every cognitive researcher to have a perfectly representative sample. Researchers may be suited best by describing the sample they are able to get and acknowledge any constraints in making generalizations. To address the lack of generality, researchers have made proposals such as Constraints on Generality (COG) statements (Simons et al., 2017). It may not be possible to make every study perfectly ecologically valid with a heterogeneous sample representative of all humanity. But we can at least acknowledge and characterize how the study may fall short. This approach is best for the collaborative process; that way, the next study may build and understand how their study and data relate to the prior work.

\section{Environment and context are underspecified}

Cognitive researchers tend to take a context-free "brain in a vat" approach. While there are some notable exceptions (see below), researchers typically attempt to control for or ignore variables deemed outside of the scope of basic cognitive processes. This controlling approach is meant to eliminate extraneous variables from consideration in order to define laws for human cognition. Controlled cognitive experiments mirror the image of how successful sciences, such as physics, seem to work.

The question for cognitive researchers is, will we be able to sufficiently characterize human behavior with the "brain in a vat" approach? This approach tends to put the participant's context in the background. What is included in context? The term is purposely broad as there are many examples of context that can affect human behavior. Examples may roughly fall under the categories of prior developmental experiences (e.g., Gunderson \& Levine, 2011; Maloney, Ramirez, Gunderson, Levine, \& Beilock, 2015; Smith \& Thelen, 2003), cultural variations in experience(Boykin \& Bailey, 2000; Markus \& Kitayama, 1991; R. Serpell \& Boykin, 1994; Z. N. Serpell, Boykin, Madhere, \& Nasim, 2006), and the immediate physical environment (Barr, 2010; Erickson \& Newman, 2017; Thelen, Schöner, Scheier, \& Smith, 2001).

The use of a context-free approach may mean that cognitive researchers don't actually end up with informative data about what they want to characterize. Because we know context, in the form of developmental experiences and culture, 
deeply affects human behavior, taking human behavior, with no information about said context, as a base for studying human cognition, is an ill-fated proposition.

The structuring of experiments to emphasize a controlling framework may actually be counterproductive to coming up with useful laws about human cognition. Human behavior may not be characterized by the elegance of the classical mechanics physics F = MA; few variables with a straightforward relationship with simplifying assumptions. Human cognition may be more likely best characterized like the more complex $-\nabla P+\nabla \cdot \sigma^{\prime}+\rho f=\rho(\partial v \partial t+v \cdot \nabla v)$ of Navier-Stokes equations of fluid dynamics; many variables, more math needed to properly characterize the complex system.

Exceptions to the context-free approach include work related to ecological psychology (e.g., Heft, 2013; Spencer, Dupree, \& Hartmann, 1997). At the least, this work alters the level of analysis from "the mind" of the participant to "the mind in context." Consider the perspective of Bronfenbrenner's Bioecological model of human development (Bronfenbrenner, 1977). This approach views humans as being in nested systems that influence behavior. Bronfenbrenner's multilevel model (see Figure 1) focuses on the levels of influence on individual behavior, from macrosystems (overarching culture) to microsystems (immediate family). This approach focuses on evaluating human behavior through multiple levels of analysis. Traditional approaches to cognition concentrate almost exclusively on the individual, with little consideration for individual differences (e.g., gender, age, health) or the broader context described by ecological systems theory.

\section{Focus on averaged behavior obscures individual and contextual variation}

Cognitive research regularly relies on quantitative characterizations of behavior that are combined across groups of participants. The approach of comparing an experimental group and control group is quite common. Statistical comparisons implemented through Analysis of Variance (ANOVA) and T-test are frequently used. How well can our results reflect behavior and cognition if we focus our analysis only on group differences, leaving potential individual and contextual variation and within the groups as noise? There is some danger that averaging across participant groups may obscure the behavioral patterns (Siegler, 1987); even if group differences are meaningful, the absence of attending to variation limits what we can explain. There is some work that does consider individual differences in cognition, using statistical approaches such as hierarchical linear modeling (McNeish, Stapleton, \& Silverman, 2017). Also some work in developmental psychology and cross-cultural psychology often attends to developmental and cultural contexts (e.g, Kuwabara \& Smith, 2012). Individual differences in related research on educational attainment and related outcomes such as intelligence testing (Conway, Kane, \& Engle, 2003; Daniele, 2021; Tourva \& Spanoudis, 2020).

While the use of experimental and control groups may be warranted, lack of attention to individual variation may limit the use of the study conclusions. In some sense, this is simply the nature of highly variable data analyses through group comparison methods such as ANOVA. For example, in some studies of cognitive 
development with infants, it is possible to show statistically that a group of participants "know" a rule, while not a single participant's data supports the conclusion that the individual does. The behavior of one group of participants may, when analyzed as a group, be different than another in a way that is meaningful. The goal of a comparison method such as ANOVA is to demonstrate between group variance is greater than within group variance. While there may be group differences, individual differences within each group may mean that it is unclear exactly how to apply overall conclusion that the participants "learned the rule" if many individuals may not have. This is not a problem limited to infant work; research on numerical cognition demonstrates that group average results do not tell us much about individual participant's numerical skills (Chesney, Bjalkebring, \& Peters, 2015).

As we can see in cross-cultural and developmental research, it is at times worth examining sub-distributions in participants behavior. Cross-cultural researchers may describe the distribution of outcomes on some task for two different cultures. Developmental researchers may describe a distribution of outcomes on a task for multiple age groups. The question becomes, rather than positing a single distribution of behavior for generic human actors, various subdistributions that depend on the developmental, cultural and societal context of the participants. This may be the best way to balance individual variation, with the influence of participants" context.

The current lack of emphasis on individual and contextual differences may reflect cognitive researchers' generality assumptions and emphasis on creating parsimonious experiments and explanations. Participants in Condition A did one thing; participants in Condition B did another thing. Reliance on group averages reflects researchers' attempts to characterize a straightforward and simple effect. While there may be some use in this sort of comparison approach for estimating treatment effects, it ignores potentially substantial individual variation and provides no information about the behavior of individual participants. Maximizing experimental elegance may severely limit the usefulness of the results of cognitive studies.

\section{Theory construction is underdeveloped}

An important aspect of assessing what is known about human cognition is the state of theories of cognition. The metascientific scholarship has addressed the relationship between empirical data and theory in cognitive research (e.g., Guest \& Martin, 2020; Muthukrishna \& Henrich, 2019; Szollosi \& Donkin, in press; van Rooij \& Baggio, 2020). While the details of the proposals vary, a common assessment is that theory building is lacking in cognitive research. This is not to say that there are not cognitive researchers interested in theory, as the authors of such papers would count themselves so. Examples of successful theories include Shepard's law (Navarro, 2021; Shepard, 1987), Marrs level of Analysis (Marr, 1982; van Rooij \& Baggio, 2021).

However, the discipline priorities focus on descriptions of behavioral effects, often without a clear link to any sort of larger well-formed theory (e.g., Guest \& Martin, 2020; Townsend, 2008). Psychological science has been described as 
"overwhelmed with things to explain, and somewhat underwhelmed by things to explain them with" (Cummins, 2000; van Rooij \& Baggio, 2020). The downside is that cognitive research may seem to be a collection of effects, of uncertain replicability and unknown generalizability, with at best loose connection to underlying theories of cognition. This makes cumulative progress for the field difficult.

\section{Potential Solutions and a way forward}

The aforementioned problems with cognitive research are broad and have engendered a fair amount of attention. I do not necessarily disagree with proposed solutions, such as using more heterogeneous participant samples. However, I contend that the concerns are inexorably connected to the basic assumptions and norms of cognitive research that have, with few exceptions, held since the cognitive revolution. A comprehensive reconsideration of the assumptions, level of analysis, the scope of consideration, and the overall approach of cognitive research may be needed in order for the field to progress. I contend that we already have many of the necessary tools and approaches to move on from cognitive universalism. The contribution of this proposal is not to create these tools, as the work of other researchers is characterized below. The goal of the current proposal is the demonstrate that this work can be brought together as a coherent framework for studying human cognition that moves beyond cognitive universalism.

In this proposal, I direct researchers of human cognition to existing tools that will improve the study of human cognition. The proposal combines approaches taken from across the social and behavioral sciences, such as sociology, social psychology, and public health. Both within cognitive research and across other behavioral fields, there are instances of researchers incorporating some aspects of the proposal. This is helpful as cognitive researchers can find examples of these approaches. However, these examples tend to be the exception and are often not seen in studies concerned with human cognition.

The proposed changes should be viewed not just at the level of an individual study but a research program and the literature in general. I recognize that researchers' goals and constraints will vary. I do not expect all cognitive studies to incorporate all of the proposed changes in the same way. The proposal is not an exact recipe to be followed. The proposal is meant to contain guideposts for what priorities are necessary if our collective goal is a useful characterization of human cognition.

The search for cognitive universals may progress best by abandoning the idea of the context invariant universals. There may be some cognitive processes that generalize to $100 \%$ of the human population, others to $95 \%$ or even $50 \%$. How do we know which is which? Especially if we are collectively working with a very small slice of the global human population? We need a way to be able to tell the difference between a cognitive result that generalizes to $100 \%, 70 \%$, or $30 \%$ of human population. A necessary first step is characterizing who our participants are in terms of developmental, cultural and societal context. This contextual space has been shown again and again to affect human behavior. As clearly seen in developmental psychology, cross-cultural psychology, and sociology. 


\section{RECONSTRUCTING COGNITION}

It is time for cognitive researchers to think through how adapt this to our research questions.

\section{Focus on Participants' Context}

Studies of cognition do not take participants' context seriously enough. Human cognition is so inexorably tied into the environmental context that it is not possible to study in isolation, as has been the dominant strategy since the Cognitive Revolution. Measures of contextual factors can be put into categories that may include; 1) demographic variables such as race or SES that may shape cognition through lived experience; 2) "non-cognitive" factors such as socio-emotional learning, motivation, anxiety, emotional regulation, personality, goal orientation, implicit self-theory as other critical individual difference variables that cannot be separated from behavior; 3 ) the immediate environment such as the specifics of behavioral testing and data. For example, is participant behavior open-ended selfreport, multiple-choice, reaction time, etc.? Are the materials written, pictures, objects, etc. These sorts of effects are not side interests to a pure measure of cognition; they are it.

Cognitive studies tend to take the approach of assuming the participants exist vacuum. Does ignoring context help researchers develop universal laws that apply to all humans across cultures? Physicists of classical mechanics can at least have access to a real-life system that resembles their model. Cognitive researchers do not have access to the human equivalent. Humans do not and cannot exist in a vacuum. They are embedded in culture and are the product of their individual developmental experiences. There is no way to separate humans from the effects of developmental and cultural contexts. It would behoove cognitive researchers to consider this reality in their approach. While there are many arguments about which aspects of context to attend to, the most important thing here is that researchers are asking the question of characterizing how human cognition is inexorably influenced by context.

Depending on the study's goals, the researchers may wish to focus on different aspects of the broader context. Studies of children's cognitive development may focus on the parental home environment. Studies in classroom settings may focus on the classroom and school details as part of the educational context. Some studies have focused on aspects of the physical environment, such as local environmental pollutants like air pollution or lead (e.g., Forns et al., 2017; PayneSturges et al., 2019).

What about a "regular" study of adult cognition? What context could be relevant? We operate under the assumption that human participants are living in a society. Their existence living in human society means we can and should attempt to measure some aspects of their prior experience and current situation in that societal context. What part of the participant's context should the researcher attempt to estimate? A context such as a student's classroom or school or something more general about that person's experience in life embedded in a specific society. Participant race, gender, and socioeconomic status are a few examples of measures. It is crucial to note that these should not be thought of as simply demographic characteristics of the individual, but an initial gateway to assessing variations in 
experience and context that we know affects human behavior. People with the same demographic characteristics can have very different experiences. A simple demographics form, while a good start, may not be enough.

Researchers will need to learn about these demographic categories and how other social scientists measure them. Race, for example, is not a biological category. There is no such thing as a "biological race." Race is a socio-political construct. This is a crucial point. It would behoove researchers to gain an understanding of the history of race as a category (e.g., Fuentes et al., 2019; D. Roberts, 2011). The way race is constructed, and the racialized experiences of participants can vary from location to location.

If race isn't "real," what should we measure? Racialized experiences that interact with the context of the participant. There are relevant measures, such as perceived discrimination (e.g., Seaton, 2010). It is worth noting how other social and behavioral sciences have addressed racialized experiences. One such example is how public health research treats racial disparities in health outcomes (e.g., Braveman, 2006; Roux, 2011). The source of these disparities is not rooted in biological essentialism regarding race. The disparities are due to environmental disparities that the people experience. Additionally, research has shown the effects of perceived racial discrimination and the risk of cognitive impairment in aging (e.g., Seaton, 2010). The mechanism of this effect is chronic stress. Related, there is work on the cognitive effects of perceived racial discrimination (Ozier, Taylor, \& Murphy, 2019). Though it should be noted that race is not the entirety of contextual factors that could be incorporated in cognitive research.

There is no reason to believe that cognition is special compared to health, education, or other biological and social outcomes. Human cognition is not somehow shielded from or independent of environmental effects, developmental experiences, culture, and other contextual factors. We know that context affects human cognition, there is not a lack of examples (see much of cross-cultural psychology and developmental psychology) and the theoretical work of other researchers (e.g., Serpell \& Boykin, 1994; Serpell, Boykin, Madhere, \& Nasim, 2006). The issue is not that we simply need to note the occasional effect of context on participants' cognition. Rather than the context-cognition interface needs to move from being an interesting side note to being the primary focus and level of analysis for cognitive researchers.

How can we do we balance an unfettered assessment of all potential relationships with the need for rigorous, confirmatory testing guided by appropriately powered designs and a priori characterization of hypotheses? That is going to be tough to do if we limit the scope to what is done in one study. Most likely the need to work on potentially exploratory analysis that contextual space will be done separately than more focused confirmatory studies. We already have some acknowledgment of the value of different types of studies. There unfortunately will not be a simple answer for which context to measure when. That is going to have to be worked out across many areas of research over many studies. Similar endeavors have begun in other social sciences such as Public Health (Palmer, Ismond, Rodriquez, \& Kaufman, 2019) and Organizational Psychology (Johns, 2018). Important Johns notes, "it's not that context has never been studied, but it 
has often been studies in a rather haphazard way". The situation in cognitive research is similar.

\section{Embrace complexity}

Certainly, since the Cognitive Revolution, the norm for cognitive research has been tightly controlled experimentation, as that seems to have been a successful approach in the natural sciences (Mandler, 2007; Núñez et al., 2019). However, this may not be the best approach for social and behavioral sciences. Instead of focusing on the controlled experiment model of physics, we might be better off taking inspiration from other fields that cannot employ experimentation in the same way, such as astronomy, public health, and other social sciences. These fields rely heavily on quantitative approaches that focus on defining relationships and non-linear dynamics between many observed and latent variables.

I propose that cognitive researchers would be better off taking a complex systems approach to statistically modeling data, rather than the typical direct comparison of experimental groups often through ANOVA and t-tests. This will give researchers a way to recognize that not all that affects the measurements can be controlled for. Suppose we combine this sort of approach with a focus on collecting contextual data and assessing individual differences. In that case, we can imagine study results that describe a complex relationship between many measured and latent variables. Some relationships may generalize across participants; others may not. The result would be a bit more complicated than merely describing the main effect in ANOVA.

There is a large set of quantitative approaches, common in social sciences, which fit well with embracing complexity-for example, structural equation modeling (SEM), hierarchical or multi-level linear modeling (McNeish et al., 2017), path analysis, and system dynamics modeling (Figure 3). In the case of HLM for example, the difference from a standard t-test or ANOVA in is that participants can be further nested within the groups. This is used to evaluate classroom effects in educational research. Nesting could be done via relevant contextual variables. The researcher can then not only see if there are differences between groups but variation within groups. By measuring multiple individual differences, the researcher can start to make inferences about the types of participants who show the biggest effect. These are not an uncommon approach across the many social sciences fields; however, they do not appear to yet be popular in cognitive studies.

Another option is to use one of the many quantitative methods under the umbrella of Complex Systems. One such approach is system dynamics, a computational modeling method to understanding the non-linear behavior of complex systems over time (Hovmand, 2014). System Dynamics has some similarities with structural equation modeling (Hovmand, 2009)

These methods may feel like a step backward as this sort of approach would invariably lead to what seem like incomplete stories. That may be better in the long run. A well-characterized incomplete story that can be followed up with another study may be more useful for the field than a complete story that is incorrect. We 
need to step away from controlled lab-based experiments with participants of unknown context as the gold standard approach to cognitive research.

\section{Employ Quantitative Intersectionality frameworks}

Intersectionality is a theoretical and analytical approach that simultaneously considers multiple categories of identity, difference, and inequality in power, such as gender, race, class, and others (Crenshaw, 1989). The idea here isn't about personal identity per se, but how it interacts with societal context (see McCormickHuhn, Warner, Settles, \& Shields, 2019). Intersectionality as an approach is often associated with qualitative work in feminist psychology and critical race theory (Cole, 2009); however, a variety of social sciences have developed approaches that employ intersectionality.

Intersectionality may seem far afield from figuring out the fundamental processes of human cognition. It is not. Researchers of human cognition almost exclusively rely on human behavior as our data for characterizing human cognition. Human behavior for each individual is embedded within specific developmental experiences and societal context. If you study human cognition using behavior, your data is affected by your participants' developmental experiences and societal context, whether you attend to it or not. Ignoring this context has not been working well for cognitive research. Ignoring this context limits cognitive research to what are essentially inadequate descriptions of cognition and keeps us from the grand characterization of human cognition that is the goal of many researchers. Embracing the intersection of human behavior and context as the correct level of analysis for cognitive research is the way forward.

How intersectional approaches may be adopted is not a trivial question and may require some shifts in perspective by cognitive researchers (McCormick-Huhn et al., 2019). Fortunately, there is some precedent for implementing intersectional approaches in a quantitative manner that may be of use to cognitive researchers. First, there is work in quantitative intersectionality in other social sciences (e.g., Bauer \& Scheim, 2019; Bécares \& Priest, 2015; Jackson \& VanderWeele, 2019; Richman \& Zucker, 2019). Secondly, researchers with expertise in quantitative methods have laid out potential methodological approaches that may fall under the general approach of quantitative intersectionality (Else-Quest \& Hyde, 2016a, 2016b).

There are many ways that quantitative analysis can integrate an intersectional approach. Components that can potentially use an intersectional framework include theory, design, sampling techniques, measurement, data analysis, interpretation, and framing (Else-Quest \& Hyde, 2016a, 2016b). Statistical analyses, for example, can focus on multiple or additive main effects along with interactions between main effects. What works best for each study may depend on the details of the scientific problem and the limitations of the available participants? The expectation is not that each cognitive researcher has a participant sample that is "perfectly balanced" per se, but that researchers attend to the context of their participants enough to know who they are. 


\section{Explicitly consider constraints on generality.}

The range of generality is an important part of interpreting research findings. A mismatch between how general the researchers believe the results to be and how well the results generalize when tested can hamper long term efforts (DeJesus, Callanan, Solis, \& Gelman, 2019). Cognitive researchers tend to work with the goal of generalizing to all humans. Some exceptions include research with child participants and research on language. In those cases, researchers may note that results may not be assumed to generalize to a different age or different language. Either way, there is still an assumed core of human cognition that is the same across all humans.

Cognitive researchers may wish to explicitly state which aspects of the reported behavior they believe will generalize. Perhaps consider how behavior may vary across different samples due to experience or other contextual factors. This may seem a bit like speculation at first; however, we should be able to collectively build better ideas of how participants' context can vary and how that may affect behavior. This can be an important part of our theories of cognition and not a side issue for those interested in "special populations."

Constraints on Generality statements can include known boundary conditions of the theory, the necessary conditions for the effect, factors that be important to behavior even if their role has not been tested, how the participant sample is or is not representative of the target population, what the target population is. What aspects of the materials and procedure are needed to produce the effect? If the materials are shown on a computer screen, do we expect the effect to generalize to materials that include physical objects? Even that cannot be assumed (Barr, 2010; Pan, Bingham, \& Bingham, 2013). Does the effect rely on cultural norms that may vary across time and place? Does the effect rely on certain assumptions about development (e.g., 'typical' development)? Often these sorts of questions are left unsaid or at most implied. They should be explicitly noted by the researcher.

This may seem like admitting defeat. How could one study have a participant sample that is representative of all humans? Such constraints do not have to mean that the study results are not useful or interesting. Consider alternatives to random sampling from the population, such as model sampling (Zhao, 2020), which allows for the assessment of inevitably imperfect samples.

\section{Construct theories aimed at both cognition and context}

The focus of cognitive research has been primarily on 'internal' cognitive processes. However, the internal cognitive processes are clearly affected by external context, including developmental experiences and culture. The range of variation may have limits, but there are far too many examples of the effect of context on cognition and behavior for us to think that the idea level of analysis for researchers of human cognition is the proverbial mind in a jar, implementing cognitive processes in a vacuum. We all know this is incorrect and limited, so why can't we replace it with a focus on a level of analysis that simultaneously considers external context and internal cognitive processes? It's hard to do. But it is likely the way forward for high verisimilitude theories of human cognition. 
Computational modeling is likely a useful tool in theory building (Guest \& Martin, 2020). There are some interesting existing theories. That might do well here, embodied cognition, dynamic systems, and other complex systems approaches. I leave it to the reader to determine exactly what theoretical positions may be adapted to integrate context on equal footing with "internal" processes. The important point is that developers of cognitive theory need to set their sights on that goal. This should affect the type of predictions, hypothesis, and data that is relevant.

Consider the prior four recommendations in concert with sifting the default level of analysis. Regardless of the topic or methods employed by the researcher, cognitive research must grapple with the interaction between cognition and context. That is where the real thing is, not some imaginary default human. I fully expect that if this recommendation and those of the full proposal are implemented, cognitive studies of the future may look very different than what is typical now and what has been done in the past.

\section{An example approach}

I include a "toy" example of a potential study set up that incorporates some of the prior proposal. Rather than just looking for a difference between Group A and Group B, we also include a wide range of variables about the participants' context. This is not information to be controlled for but is integrated as part of the overall model. While this single study may incorporate some of the proposal, it is important to view the proposal not as a recipe for a single study, but a set of priorities for the field. Some aspects are complex and will need to be worked out over multiple studies, perhaps through the use of multi-lab collaborations, such as Psych Accelerator (Moshontz et al., 2018).

The example study focuses on the role of executive function in math learning. The study set up is similar to much cognitive work in that it primarily involves measures of participant behavior on experimenter designed tasks. Several aspects of the study design implement aspects of the current proposal. First, consider the selection of study participants. Recruitment is focused on helping provide a sample that is heterogonous on some demographic variables while oversampling on others. This may require more extensive recruitment efforts. In this study, our goal is to oversample our target population, African American children, from $3^{\text {rd }}$ to $5^{\text {th }}$ grade. Second, participant measures include extensive demographic information, age, race, gender, and socioeconomic status measures. We also include relevant questionnaire items about participants' experiences, including the home environment and school environment. Rather than develop our own measures, we are able to use measures developed by social scientists in other areas, such as social psychology and educational psychology. This required reading outside our home discipline and was greatly helped by collaboration with colleagues outside of cognitive research. This is how we develop a measure of the participant's context. In this study, we are particularly interested in how well students perform on math tasks in a classroom environment. Thus, we include questions about each student's school as part of developing their context measure. 
Lastly is the development of a quantitative model of these variables. Suppose we think of this as almost a system dynamics approach. In that case, there are the participant context variables, measured behavior on the cognitive tasks of interests, characterization of the experiment including details of the cognitive task, and participant condition, if applicable, latent cognitive variables such as executive functioning or numeracy.

In this example, we can visualize the results as part of a 3-dimensional matrix (see Figure 4). Matrix dimensions correspond to the following, 1) the different tasks or measures or constructs, 2) different conditions in the study, 3) varying context of the participants. The matrix values correspond to some quantitative measures of behavior on the task. For a math task, it could be as simple as percent correct or reaction time.

The data summarized in the experimental matrix is used as the input for a multigroup HLM. Contextual variables can be used to set participant groups. We might use an HLM model to evaluate the differing effects of context and experimental condition on participants' behavior. Similarly, a Path (e.g., Alwin \& Hauser, 1975; Edwards \& Lambert, 2007) analysis and latent class analysis (e.g., Nylund-Gibson \& Choi, 2018) could be used to evaluate the relation of multiple contextual variables on the target outcome. Contextual variables can be thought of as covariates and interactions. This sort of setup is not unusual in some social sciences. (Figure 3).

In terms of writing out the study conclusions based on HLM or path analysis results. A multifaceted relationship between multiple variables may or may not be easily broken down into a simple story. This may lead to results that appear to have more caveats, "executive function matter for math, more so for certain tasks, and in certain contexts, with some individual variation.". This does allow the researchers to build up an understanding of the relationship between cognition and context. Though the emphasis in this proposal has been to focus on variation, we do not expect the range of behaviors on cognitive tasks to be so variable as to be intractable. The researcher will likely develop an idea of the range of behaviors and how they relate to the range of contexts. Far from being an "anything goes in cognition," we expect that constraints in context will naturally align with constraints in cognitive processes.

\section{Potential Difficulties}

The proposed approach is not without its difficulties. This may seem like a potentially never-ending push to measure more and more participant context. One potential issue is that the proposal is just too much to consider at once. It probably is. How much is enough for one study to address? It is not likely the case that every potential context measure will affect behavior on cognitive tasks. However, the current state of mostly ignoring context is untenable. Expectations need not be that every individual study will address all potential effects of participant context on behavior. It would still be useful for the field for the median cognitive study to integrate variations in participant context far more than is the current norm. 
The reader may ask, is this really cognition? Is bringing all these seemingly extraneous variables in really studying cognition? Are we focusing on outcomes and not mechanisms? Yes, it is clear from the literature noted in this paper, and elsewhere, these variables are already here affecting behavior. It is up to the researcher to build their models and theories of human cognition with or without them.

The ultimate conflict is between an approach that treats behavior as primarily due to inherent individual characteristics, as opposed to a dynamic interaction between that individual and their environment. That is, considering individual differences without simultaneously attending to differences in context is fundamentally inadequate.

Will the incorporation of race lead to the reification of so-called racial differences? This is a serious question-particularly given psychology's extensive history of supporting scientific racism (Winston, 2020). Attending to race does not have to support racism. Especially because what we really want to attend to is societal-context. Participant's racialized experiences are not a characteristic of themselves individually, so much of the societal context (McLoyd \& Randolphh, 1984). Also, keep in mind that race is not, and has never been, a biological category (e.g., Roberts, 2011).

Is adopting an intersectional framework necessary? Using intersectionality as an approach, be it quantitative, qualitative, or both, would be in line with other social and behavioral sciences. Despite barriers, there have been calls for the use of intersectional frameworks in clinical psychology (Banks \& Kohn-Wood, 2002), developmental psychology(Syed, Santos, Yoo, \& Juang, 2018), social psychology (Bowleg, 2017), personality psychology (Sabik, 2016), and industrial and organizational psychology(Sanchez-Hucles \& Davis, 2010). While the idea may initially seem far afield from relevance to cognition, it is integral in understanding how to characterize participants' context. Useful explanations of how intersectional approaches can be adopted in quantitative methods exist (Else-Quest \& Hyde, $2016 a, 2016 b)$, and there is no reason they cannot be integrated into studies of cognition.

\section{Conclusion}

The current proposal emphasizes the important theoretical point: it has been difficult to characterize some sort of default human cognition that generalizes across the entire species because there isn't one. Or, at the very least, cognition is contingent on development, culture, and context is far more than we as a field have considered. The sooner the field lets go of the assumption of normative cognition and move forward with a more complex and real-world grounded idea of how cognition can be characterized, the better. While we have certainly gotten to the point where many would agree that the "brain in a jar" perspective is not sufficient, we have failed to consider the exterior context sufficiently. As humans are embedded in social contexts, and of course, developmental environment matters, we need to increase the focus on a characterization of the context of participants in our cognitive studies. 
If it seems like I am suggesting that researchers of human cognition become a bit more like sociologists, I am. The proper level of analysis for a comprehensive understanding of human cognition needs to be nudged in the direction of the domain of sociology. Sociology is primarily focused on the social systems that humans make up and live in. While not the primary focus on cognitive research, it does not seem that ignoring the social systems is useful in characterizing human cognition. Cognitive researchers need not abandon their craft, so much as reconstruct it by expanding their methods and theoretical considerations. This will have the additional benefit of cementing connections between cognitive researchers and researchers across allied fields.

Our intention is not to be overly prescriptive. Context is meant to be a broad term to give the researcher leeway in defining what is relevant context. To some degree, this must be a communal endeavor and will not be one that any individual researcher or research team will determine. Defining how to integrate participants' context into cognitive studies will be difficult but necessary work. Some researchers have already begun, but more is needed. Reimagining our collective approach to studying human cognition will have challenges. However, the evidence is clear that the status quo approach is limited at best. The discipline may have to commit to serious consideration of a change in our collective approach or anticipate a place on the shelf next to phrenology.

\section{Figures}

Figure 1. Bronfenbrenner's Ecological Model. A framework for human development. The individual is embedded in and influences by multiple levels of the external environment.

Figure 2. Muller-Lyer illusion. The horizontal lines are perceived as being different lengths despite being the same. This effect is seen in WEIRD population samples, but the effect of smaller magnitude or absent for others.

Figure 3. Example diagram of a Path analysis. In this example, exogenous variables 1-5 relate to three endogenous variables. For example, variables could be executive function and numeracy, which then predict academic achievement. Many potential paths can be hypothesized and evaluated.

Figure 4. Experimental matrix. Matrix dimensions correspond to Participant context $(\mathrm{Y})$, Conditional variation $(\mathrm{X})$, and Task measures $(\mathrm{Z})$.

\section{References}

SRCD policy (January 2020) https://www.srcd.org/news/new-sociocultural-policyenacted-across-all-srcd-journals 
Center for Open Science. COS mission[Internet]. Charlottesville, VA: The Center [cited 18 Nov 2016].<https://cos.io/about_mission/>.

NIH policy on the inclusion of women and minorities as subjects in clinical research. NIH notice no. NOT-OD-18-014 .(https://grants.nih.gov/policy/inclusion/womenand-minorities.htm

Alwin, D., \& Hauser, R. (1975). The Decomposition of Effects in Path Analysis. American Sociological Review, 40(1), 37-47. https://doi.org/10.1088/17518113/44/8/085201

Banaji, M. R., \& Crowder, R. G. (1989). The Bankruptcy of Everyday Memory. American Psychologist, 44(9), 1185-1193. https://doi.org/10.1037/0003066X.44.9.1185

Banks, K. H., \& Kohn-Wood, L. P. (2002). Gender, ethnicity and depression: Intersectionality in mental health research with African American women. African American Research Perspectives, 174.

Barnes, L. L., Lewis, T. T., Begeny, C. T., Yu, L., Bennett, D. A., \& Wilson, R. S. (2012). Perceived discrimination and cognition in older African Americans. Journal of the International Neuropsychological Society, 18(5), 856-865. https://doi.org/10.1017/S1355617712000628

Barr, R. (2010). Transfer of learning between 2D and 3D sources during infancy: Informing theory and practice. Developmental Review, 30(2), 128-154. https://doi.org/10.1038/jid.2014.371

Bauer, G. R., \& Scheim, A. I. (2019). Methods for analytic intercategorical intersectionality in quantitative research: Discrimination as a mediator of health inequalities. Social Science and Medicine, 226(December 2018), 236-245. https://doi.org/10.1016/j.socscimed.2018.12.015

Bécares, L., \& Priest, N. (2015). Understanding the influence of race/ethnicity, gender, and class on inequalities in academic and non-academic outcomes among Eighth-Grade Students: Findings from an intersectionality approach. PLoS ONE, 10(10), 1-17. https://doi.org/10.1371/journal.pone.0141363

Bowleg, L. (2017). Intersectionality: An Underutilized but Essential Theoretical Framework for Social Psychology. In B. Gough (Ed.), The Palgrave Handbook of Critical Social Psychology (pp. 165-183). https://doi.org/10.1057/978-1-13751018-1_9

Boykin, A. W., \& Bailey, C. T. (2000). The role of cultural factors in school relevant cognitive functioning: Synthesis of findings on cultural contexts, cultural orientations, and individual differences., (42), 2-51.

Braveman, P. (2006). Health disparities and health equity: Concepts and measurement. Annual Review of Public Health, 27, 167-194. https://doi.org/10.1146/annurev.publhealth.27.021405.102103 
Bronfenbrenner, U. (1977). Toward an Experimental Ecology of Human Development. American Psychologist, 32, 513-531. https://doi.org/10.14195/0870-4147-48-1

Buhrmester, M., Kwang, T., \& Gosling, S. D. (2011). Amazon's Mechanical Turk: A New Source of Inexpensive, Yet High-Quality, Data? Perspectives on Psychological Science, 6(1), 3-5. https://doi.org/10.1177/1745691610393980

Cheon, B. K., Melani, I., \& Hong, Y. Y. (2020). How USA-Centric Is Psychology? An Archival Study of Implicit Assumptions of Generalizability of Findings to Human Nature Based on Origins of Study Samples. Social Psychological and Personality Science, 11(7), 928-937. https://doi.org/10.1177/1948550620927269

Chesney, D., Bjalkebring, P., \& Peters, E. (2015). How to estimate how well people estimate : Evaluating measures of individual differences in the approximate number system, 2781-2802. https://doi.org/10.3758/s13414-015-0974-6

Chua, H. F., Boland, J. E., \& Nisbett, R. E. (2005). Cultural variation in eye movements during scene perception. Proceedings of the National Academy of Sciences of the United States of America, 102(35), 12629-12633. https://doi.org/10.1073/pnas.0506162102

Cole, E. R. (2009). Intersectionality and Research in Psychology. American Psychologist, 64(3), 170-180. https://doi.org/10.1037/a0014564

Conway, A. R. A., Kane, M. J., \& Engle, R. W. (2003). Working memory capacity and its relation to general intelligence. Trends in Cognitive Sciences, 7(12), 547-552. https://doi.org/10.1016/j.tics.2003.10.005

Crenshaw, K. (1989). Demarginalizing the Intersection of Race and Sex: A Black Feminist Critique of Antidiscrimination Doctrine, Feminist Theory and Antiracist Politics. University of Chicago LEgal Forum, 1989(1), 139-167. https://doi.org/10.4324/9781315631011-38

Cronbach, L. (1957). The Two Disciplines of Scientific Psychology. American Psychologist, 12, 671-684. https://doi.org/10.1007/s10440-009-9506-5

Cummins, R. (2000). How Does it work vs. what are the laws. Two conceptions o psychological explanation. In F. Keil \& R. Wilson (Eds.), Explanation and cognition (pp. 117-145). Cambridge, MA: MIT Press.

Daniele, V. (2021). Socioeconomic inequality and regional disparities in educational achievement: The role of relative poverty. Intelligence, 84(October 2020), 101515. https://doi.org/10.1016/j.intell.2020.101515

DeJesus, J. M., Callanan, M. A., Solis, G., \& Gelman, S. A. (2019). Generic language in scientific communication. Proceedings of the National Academy of Sciences of the United States of America, 116(37), 18370-18377. https://doi.org/10.1073/pnas.1817706116

Devezer, B., Navarro, D. J., Vandekerckhove, J., \& Buzbas, E. O. (2020). The case for 
formal methodology in scientific reform. BioRxiv, 2020.04.26.048306. https://doi.org/10.1101/2020.04.26.048306

Dienes, Z. (2011). Bayesian Versus Orthodox Statistics: Which Side Are You On? Perspectives on Psychological Science, 6(3), 274-290. https://doi.org/10.1177/1745691611406920

Edwards, J. R., \& Lambert, L. S. (2007). Methods for integrating moderation and mediation: A general analytical framework using moderated path analysis. Psychological Methods, 12(1), 1-22. https://doi.org/10.1037/1082-989X.12.1.1

Else-Quest, N. M., \& Hyde, J. S. (2016a). Intersectionality in Quantitative Psychological Research: I. Theoretical and Epistemological Issues. Psychology of Women Quarterly, 40(2), 155-170. https://doi.org/10.1177/0361684316629797

Else-Quest, N. M., \& Hyde, J. S. (2016b). Intersectionality in Quantitative Psychological Research: II. Methods and Techniques. Psychology of Women Quarterly, 40(3), 319-336. https://doi.org/10.1177/0361684316647953

Erickson, L. C., \& Newman, R. S. (2017). Influences of Background Noise on Infants and Children. Current Directions in Psychological Science, 26(5), 451-457. https://doi.org/10.1177/0963721417709087

Forns, J., Dadvand, P., Esnaola, M., Alvarez-Pedrerol, M., López-Vicente, M., GarciaEsteban, R., ... Sunyer, J. (2017). Longitudinal association between air pollution exposure at school and cognitive development in school children over a period of 3.5 years. Environmental Research, 159(August), 416-421. https://doi.org/10.1016/j.envres.2017.08.031

Frank, M. C., Bergmann, C., Cristia, A., Floccia, C., Hamlin, J. K., \& Sullivan, J. (2017). A collaborative Approach to Infant Research. Infancy, 22(4), 421-435. https://doi.org/10.1111/infa.12182.A

Fuentes, A., Ackermann, R. R., Athreya, S., Bolnick, D., Lasisi, T., Lee, S. H., ... Nelson, R. (2019). AAPA Statement on Race and Racism. American Journal of Physical Anthropology, 169(3), 400-402. https://doi.org/10.1002/ajpa.23882

Guest, O., \& Martin, A. (2020). How computational modeling can force theory building in psychological science. https://doi.org/10.31234/osf.io/rybh9

Gunderson, E. A., \& Levine, S. C. (2011). Some types of parent number talk count more than others: Relations between parents' input and children's cardinalnumber knowledge. Developmental Science, 14(5), 1021-1032. https://doi.org/10.1111/j.1467-7687.2011.01050.x

Heft, H. (2013). An Ecological Approach to Psychology. Review of General Psychology, 17(2), 162-167. https://doi.org/10.1037/a0032928

Henrich, J., Heine, S. J., \& Norenzayan, A. (2010). The weirdest people in the world? Behavioral and Brain Sciences, 33(2-3), 61-83. https://doi.org/10.1017/S0140525X0999152X 
Hovmand, P. S. (2014). Community Based Systems Dynamics. Springer.

Hovmand, Peter S. (2009). Analyzing dynamic systems: a comparison of structural equation modeling and system dynamics modeling. Structural Equation Modeling, 212-234. https://doi.org/10.1017/cbo9780511542138.010

Jackson, J. W., \& VanderWeele, T. J. (2019). Intersectional decomposition analysis with differential exposure, effects, and construct. Social Science and Medicine, 226(January), 254-259. https://doi.org/10.1016/j.socscimed.2019.01.033

Jenkins, J. J. (1979). Four Points to Remember: A Tetrahedral Model of Memory Experiments. In Levels of Processing in Human Memory. Psychology Press.

Johns, G. (2018). Advances in the treatment of context in organizational research. Annual Review of Organizational Psychology and Organizational Behavior, 5, 2146. https://doi.org/10.1146/annurev-orgpsych-032117-104406

Kitayama, S., Duffy, S., Kawamura, T., \& Larsen, J. T. (2003). Perceiving an Object and Its Context in Different Cultures: A Cultural Look at New Look. Psychological Science, 14(3), 201-206. https://doi.org/10.1111/1467-9280.02432

Kuwabara, M., \& Smith, L. B. (2012). Cross-cultural differences in cognitive development: Attention to relations and objects. Journal of Experimental Child Psychology, 113(1), 20-35. https://doi.org/10.1016/j.jecp.2012.04.009

Lieberman, D. E., Venkadesan, M., Werbel, W. A., Daoud, A. I., Dandrea, S., Davis, I. S., ... Pitsiladis, Y. (2010). Foot strike patterns and collision forces in habitually barefoot versus shod runners. Nature, 463(7280), 531-535. https://doi.org/10.1038/nature08723

Maloney, E. A., Ramirez, G., Gunderson, E. A., Levine, S. C., \& Beilock, S. L. (2015). Intergenerational effects of parents' math anxiety on children's math achievement and anxiety. Psychological Science, 26(9), 1480-1488. https://doi.org/10.1177/0956797615592630

Mandler, G. (2007). A History of Modern Experimental Psychology. Journal of Chemical Information and Modeling. https://doi.org/10.1017/CB09781107415324.004

Markus, H. R., \& Kitayama, S. (1991). Culture and the self: Implications for cognition, emotion, and motivation. Psychological Review, 98(2), 264. https://doi.org/10.1037//0033-295x.98.2.224

Marr, D. (1982). Vision: A computational investigation into the human representation and processing of visual information.

Martone, M. E., Garcia-Castro, A., \& VandenBos, G. R. (2018). Data Sharing in Psychology. American Psychologist, 73(2), 111-125. https://doi.org/10.1037/amp0000242

McCormick-Huhn, K., Warner, L. R., Settles, I. H., \& Shields, S. A. (2019). What If Psychology Took Intersectionality Seriously? Changing How Psychologists 
Think About Participants. Psychology of Women Quarterly, 43(4), 445-456. https://doi.org/10.1177/0361684319866430

McLoyd, V. C., \& Randolphh, S. M. (1984). The conduct and publication of research on afro-american children: A content analysis. Human Development, 27(2), 6575. https://doi.org/10.1159/000272904

McNeish, D., Stapleton, L. M., \& Silverman, R. D. (2017). On the unnecessary ubiquity of hierarchical linear modeling. Psychological Methods, 22(1), 114-140. https://doi.org/10.1037/met0000078

Moshontz, H., Campbell, L., Ebersole, C. R., IJzerman, H., Urry, H. L., Forscher, P. S., ... Chartier, C. R. (2018). The Psychological Science Accelerator: Advancing Psychology Through a Distributed Collaborative Network. Advances in Methods and Practices in Psychological Science, 1(4), 501-515. https://doi.org/10.1177/2515245918797607

Muthukrishna, M., \& Henrich, J. (2019). A problem in theory. Nature Human Behaviour (Vol. 3). https://doi.org/10.1038/s41562-018-0522-1

Navarro, D. J. (2021). If Mathematical Psychology Did Not Exist We Might Need to Invent It: A Comment on Theory Building in Psychology. Perspectives on Psychological Science. https://doi.org/10.1177/1745691620974769

Neisser, U. (1978). Memory: What are the important questions? In M. Gruneberg, P. E. Morris, \& R. N. Sykes (Eds.), Practical Aspects of Memory (pp. 3-24). London: Academic Press.

Newell, A. (1973). You can't play 20 questions with nature and win. In Visual Information Processing.

Nosek, B. A., Ebersole, C. R., DeHaven, A. C., \& Mellor, D. T. (2018). The preregistration revolution. Proceedings of the National Academy of Sciences, 2017, 201708274. https://doi.org/10.1073/pnas.1708274114

Núñez, R., Allen, M., Gao, R., Miller Rigoli, C., Relaford-Doyle, J., \& Semenuks, A. (2019). What happened to cognitive science? Nature Human Behaviour, 3(8), 782-791. https://doi.org/10.1038/s41562-019-0626-2

Nylund-Gibson, K., \& Choi, A. Y. (2018). Ten frequently asked questions about latent class analysis. Translational Issues in Psychological Science, 4(4), 440-461. https://doi.org/10.1037/tps0000176

Ozier, E. M., Taylor, V. J., \& Murphy, M. C. (2019). The Cognitive Effects of Experiencing and Observing Subtle Racial Discrimination. Journal of Social Issues, 75(4), 1087-1115. https://doi.org/10.1111/josi.12349

Palmer, R. C., Ismond, D., Rodriquez, E. J., \& Kaufman, J. S. (2019). Social Determinants of Health: Future Directions for Health Disparities Research. American Journal of Public Health, 109(S1), S70-S71. https://doi.org/10.2105/AJPH.2019.304964 
Pan, J. S., Bingham, N., \& Bingham, G. P. (2013). Embodied memory: Effective and stable perception by combining optic flow and image structure. Journal of Experimental Psychology: Human Perception and Performance, 39(6), 16381651. https://doi.org/10.1037/a0032070

Payne-Sturges, D. C., Marty, M. A., Perera, F., Miller, M. D., Swanson, M., Ellickson, K., ... Hertz-Picciotto, I. (2019). Healthy Air, Healthy Brains: Advancing Air Pollution Policy to Protect Children's Health. American Journal of Public Health, e1-e5. https://doi.org/10.2105/AJPH.2018.304902

Rad, M. S., Martingano, A. J., \& Ginges, J. (2018). Toward a psychology of Homo sapiens: Making psychological science more representative of the human population. Proceedings of the National Academy of Sciences of the United States of America, 115(45), 11401-11405. https://doi.org/10.1073/pnas.1721165115

Richman, L. S., \& Zucker, A. N. (2019). Quantifying intersectionality: An important advancement for health inequality research. Social Science and Medicine, 226(December 2018), 246-248.

https://doi.org/10.1016/j.socscimed.2019.01.036

Roberts, D. (2011). Fatal Invention: How Science, Politics, and Big Business Recreate Race in the Twenty-first Century. The New Press.

Roberts, S. O., Bareket-Shavit, C., Dollins, F. A., Goldie, P. D., \& Mortenson, E. (2020). Racial Inequality in Psychological Research: Trends of the Past and Recommendations for the Future. Perspectives on Psychological Science, 15(6), 1295-1309. https://doi.org/10.1177/1745691620927709

Roediger, H. L. (2008). Relativity of remembering: Why the laws of memory vanished. Annual Review of Psychology, 59, 225-254. https://doi.org/10.1146/annurev.psych.57.102904.190139

Roux, A. V. D. (2011). Complex systems thinking and current impasses in health disparities research. American Journal of Public Health, 101(9), 1627-1634. https://doi.org/10.2105/AJPH.2011.300149

Rowley, S. J., \& Camacho, T. C. (2015). Increasing Diversity in Cognitive Developmental Research: Issues and Solutions. Journal of Cognition and Development, 16(5), 683-692. https://doi.org/10.1080/15248372.2014.976224

Sabik, N. (2016). Digging Deeper: Research Practices and Recommendations for Exploring Intersectionality and Social and Cultural Influences on Personality, Identity, and Well-Being. In Feminist Perspectives on Building a Better Psychological Science of Gender (pp. 143-160).

Sanchez-Hucles, J. V., \& Davis, D. D. (2010). Women and women of color in leadership: Complexity, identity, and intersectionality. American Psychologist, 65(3), 171-181. 
Seaton, E. K. (2010). The influence of cognitive development and perceived racial discrimination on the psychological well-being of African American youth. Journal of Youth and Adolescence, 39(6), 694-703. https://doi.org/10.1007/s10964-009-9438-4

Segall, M. H., Campbell, D., \& Herskovits, M. J. (1966). The influence of culture on visual perception. Bobbs-Merrill.

Serpell, R., \& Boykin, A. W. (1994). Cultural Dimensions of Cognition: A Multiplex, Dynamic System of Constraings and Possibilities. In Thinking and Problem Solving (pp. 368-408).

Serpell, Z. N., Boykin, A. W., Madhere, S., \& Nasim, A. (2006). The significance of contextual factors in African American students' transfer of learning. Journal of Black Psychology, 32(4), 418-441. https://doi.org/10.1177/0095798406292466

Shepard, R. N. (1987). Toward a Universal Law of Generalization for Psychological Science. Science, 237(4820), 1317-1323.

Siegler, R. S. (1987). The Perils of Averaging Data of Stratagies. Journal of Experimental Psychology. General, 116(3), 250-264.

Simons, D. J., Shoda, Y., \& Lindsay, D. S. (2017). Constraints on Generality (COG): A Proposed Addition to All Empirical Papers. Perspectives on Psychological Science, 12(6), 1123-1128. https://doi.org/10.1177/1745691617708630

Smith, L. B., \& Thelen, E. (2003). Development as a dynamic system, 7(8), 343-348. https://doi.org/10.1016/S1364-6613(03)00156-6

Spencer, M. B., Dupree, D., \& Hartmann, T. (1997). A Phenomenological Variant of Ecological Systems Theory (PVEST): A self-organization perspective in context. Development and Psychopathology, 9(4), 817-833. https://doi.org/10.1017/s0954579497001454

Syed, M., Santos, C., Yoo, H. C., \& Juang, L. P. (2018). Invisibility of racial/ethnic minorities in developmental science: Implications for research and institutional practices. American Psychologist, 73(6), 812-826. https://doi.org/10.1037/amp0000294

Szollosi, A., \& Donkin, C. (n.d.). Arrested theory development: The misguided distinction between exploratory and confirmatory research. Perspectives on Psychological Science. https://doi.org/10.31234/osf.io/suzej

Thelen, E., Schöner, G., Scheier, C., \& Smith, L. B. (2001). The dynamics of embodiment: a field theory of infant perseverative reaching. The Behavioral and Brain Sciences, 24(1), 1-34; discussion 34-86.

Tourva, A., \& Spanoudis, G. (2020). Speed of processing, control of processing, working memory and crystallized and fluid intelligence: Evidence for a developmental cascade. Intelligence, 83(October), 101503.

https://doi.org/10.1016/j.intell.2020.101503 


\section{RECONSTRUCTING COGNITION}

Townsend, J. T. (2008). Mathematical psychology: Prospects for the 21st century: A guest editorial. Journal of Mathematical Psychology, 52(5), 269-280. https://doi.org/10.1016/j.jmp.2008.05.001

van Rooij, I., \& Baggio, G. (2020). Theory before the test: How to build highverisimilitude explanatory theories in psychological science. https://doi.org/10.31234/osf.io/7qbpr

van Rooij, I., \& Baggio, G. (2021). Theory Before the Test: How to Build HighVerisimilitude Explanatory Theories in Psychological Science. Perspectives on Psychological Science. https://doi.org/10.1177/1745691620970604

Winston, A. S. (2020). Scientific Racism and North American Psychology. Oxford Research Encyclopedia of Psychology, (May), 1-26. https://doi.org/10.1093/acrefore/9780190236557.013.516

Young, J. Q., Thakker, K., John, M., Friedman, K., Sugarman, R., Sewell, J. L., \& O'Sullivan, P. S. (2021). Features of the learner, task, and instructional environment that predict cognitive load types during patient handoffs: Implications for instruction. Applied Cognitive Psychology, (January), 1-10. https://doi.org/10.1002/acp.3803

Zhao, K. (2020). Sample representation in the social sciences. Synthese, (March). https://doi.org/10.1007/s11229-020-02621-3 
Figure 1. Bronfenbrenner's Ecological Model

Figure 2. Muller-Lyer illusion

Figure 3. Path Analysis

Figure 4. Three dimensional data matrix 


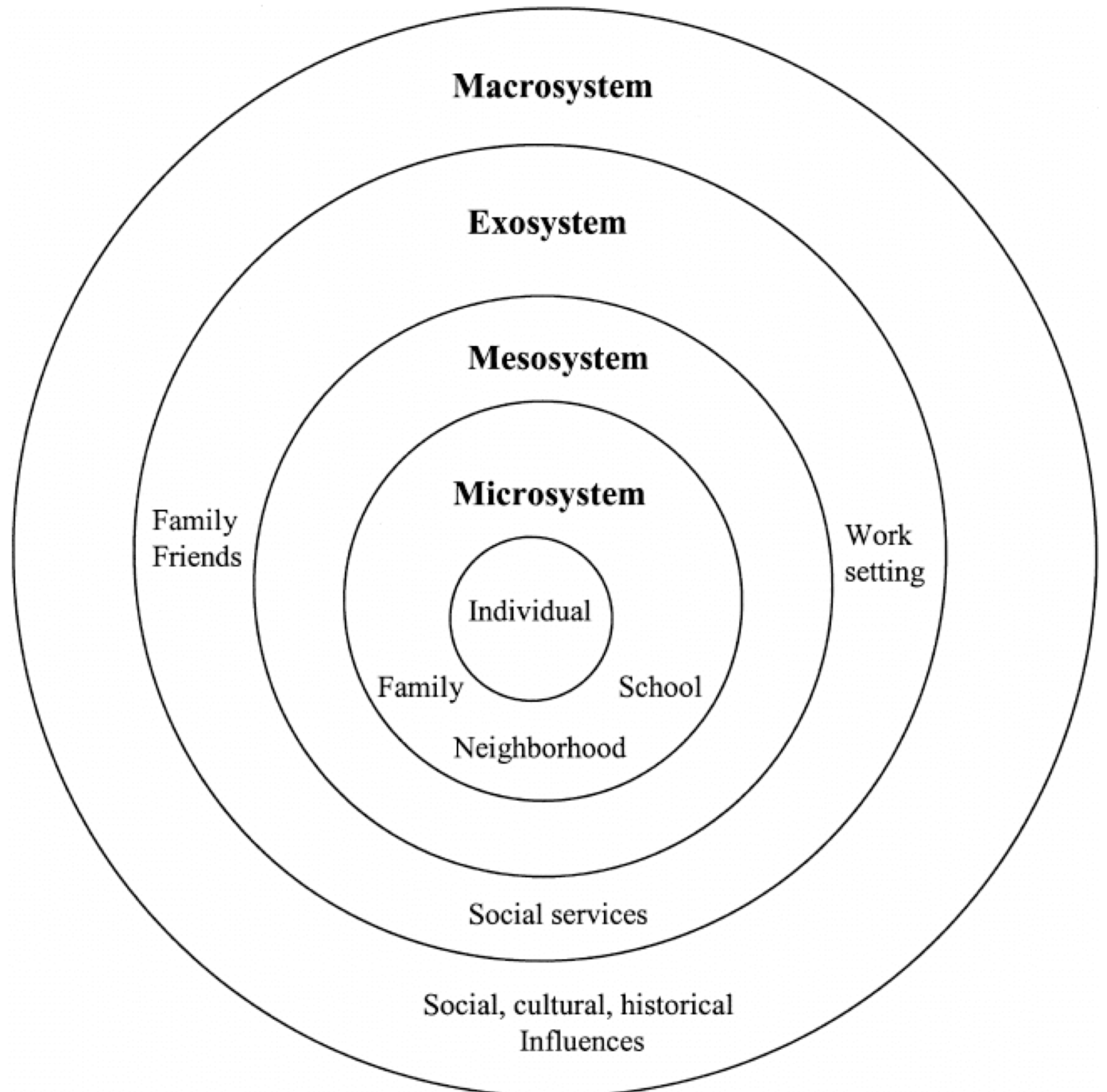



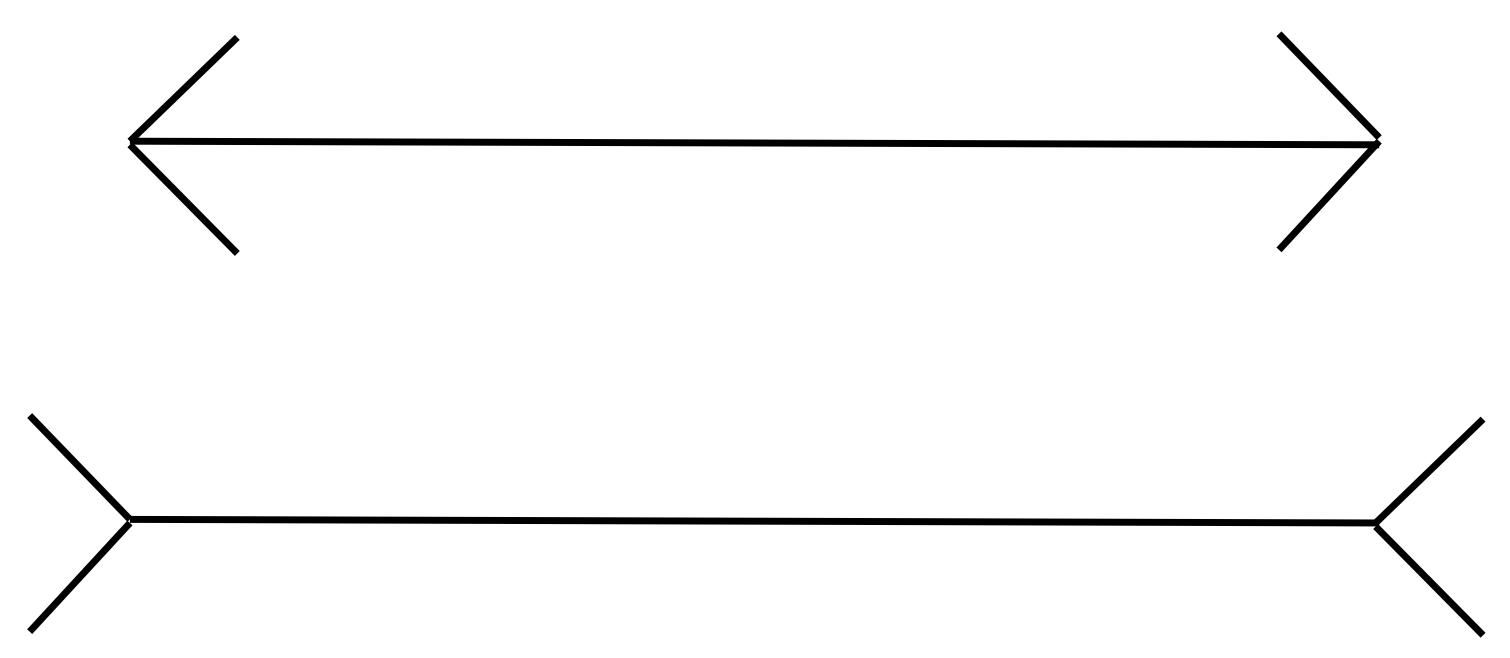


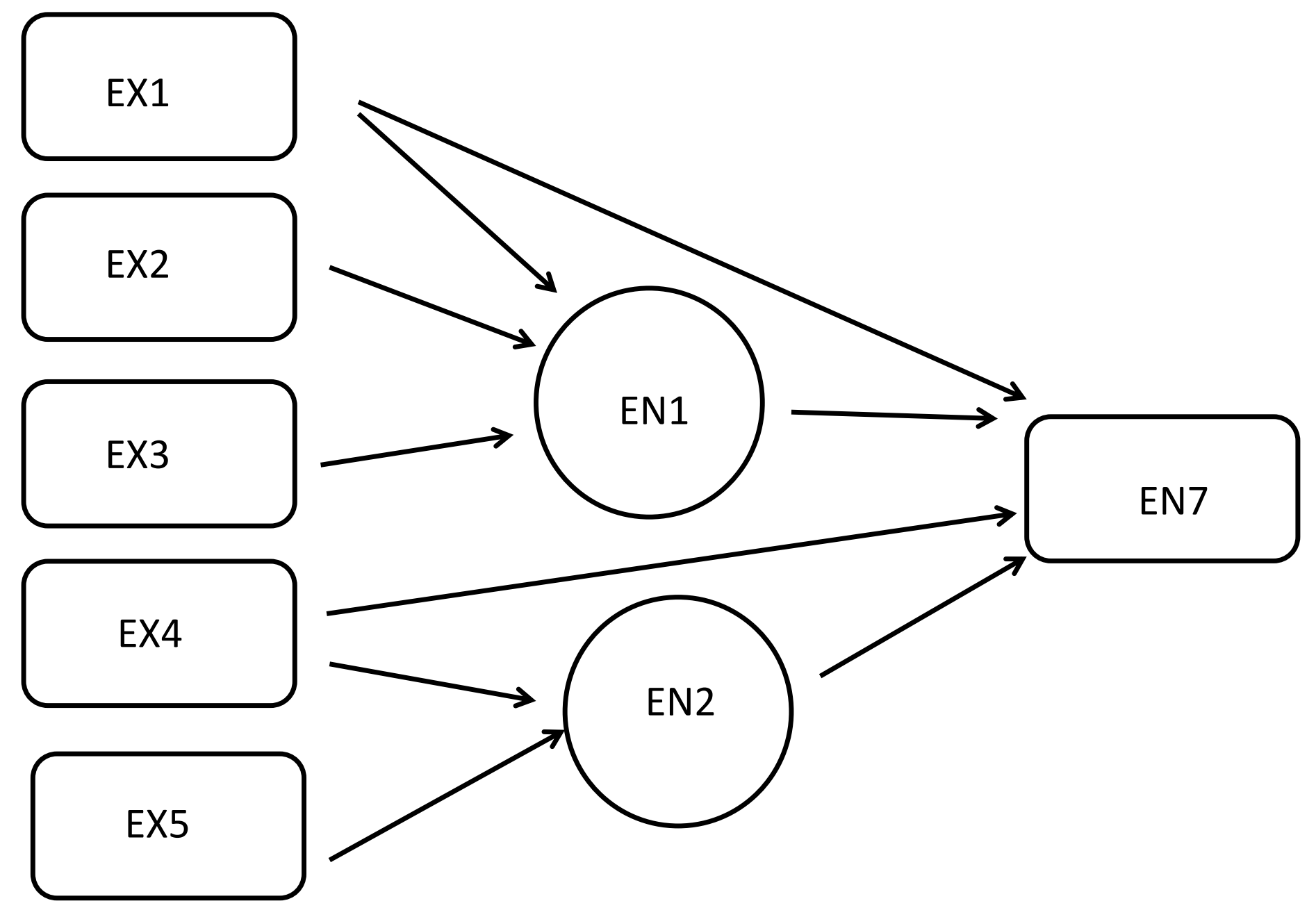




\section{Data chart for SEM model}

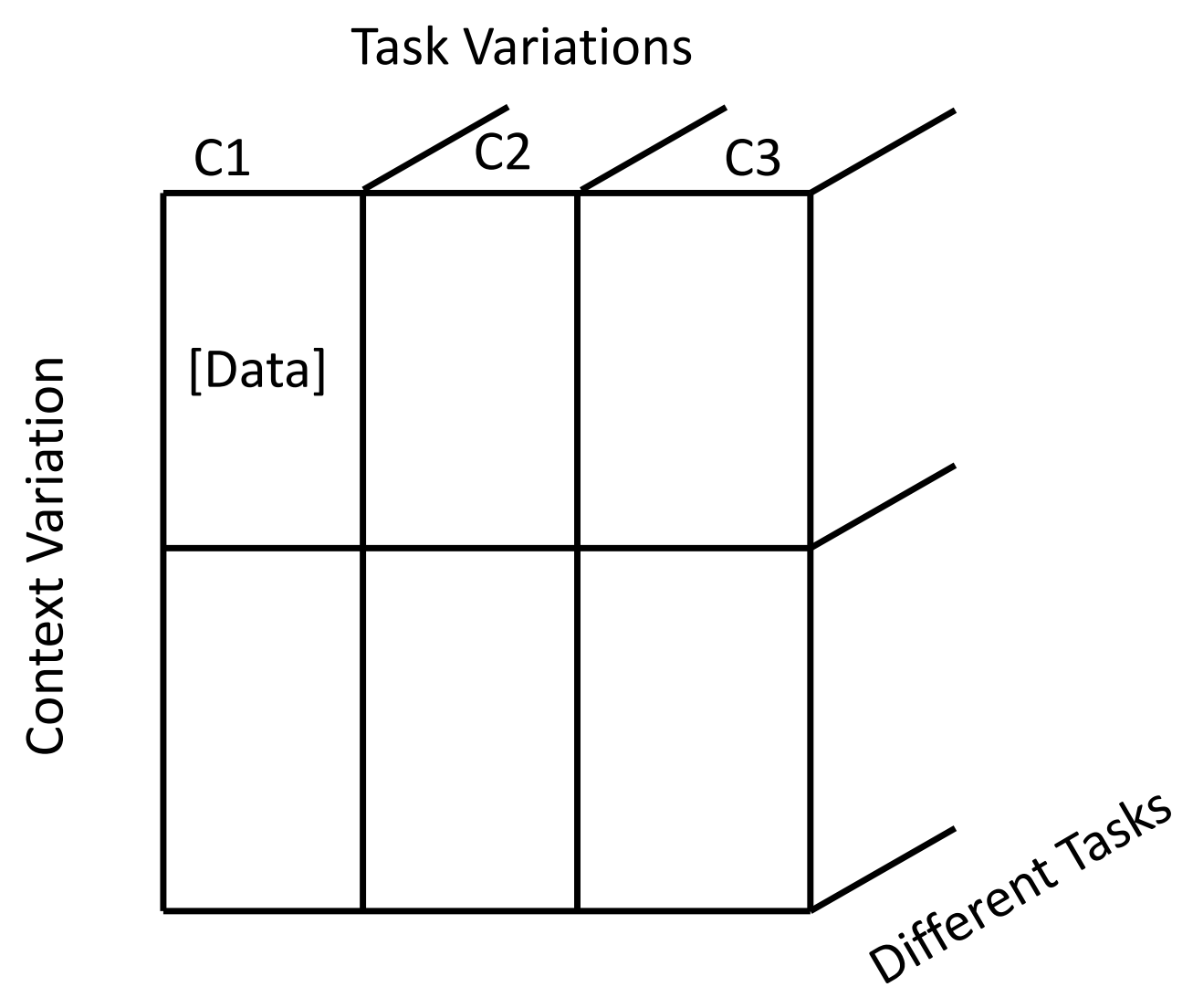

